\title{
Method for Selecting the Optimal Solution for the Design of the Gear Train
}

\author{
Fajraoui Ayoub ${ }^{1}$ and Mehdi Kamel ${ }^{2}$ \\ 1. URMSSDT, Engineering National High School of Tunis (ENSIT), University of Tunis (UT), 5 Avenue Taha Hussein P.B.56 Bab \\ Mnara 1008 Tunis, Tunisia \\ 2. URMSSDT, Preparatory Institute for Engineering Studies El Manar (IPEIMa), University of Tunis El Manar (UTM), PB.24, 2092 \\ Tunis, Tunisia
}

\begin{abstract}
Some researchers in mechanical engineering have developed systems for the design of gear transmission boxes, but almost no calculation methods exist for widespread synthesis. In this article, we outline methods for automatic determination of toothed helical gear trains and the selection criteria for the optimal choice of gear trains. In this work two methods were applied. As first design to use an expert system for the design and then optimize the design that is why we used Kappa PC and Catia for CAD.
\end{abstract}

Key words: Support systems for decision, gear train design, conceptual analysis.

\section{Introduction}

The gear transmission is the body par excellence. It perfectly meets the requirements of performance, accuracy and specific power imposed in mechanical architectures modern.

The gears are the most used component in mechanical systems of communication, and they cover many applications. Therefore, methods and software are designed for the calculation of gears. Therefore, there is a large amount of design parameters when a gear train is designed [1].

Traditional conceptions of these gear trains choices of parameters are essentially based on the experimental formula and request the diagram and are complex and redundant.

Sometimes the type of design may not be an optimal solution for the choice of optimal values, does not meet the requirements an optimization objective and cannot give consideration to all optimization goals.

But, thanks to software design computer assisted many steps are simplified and the design variables meet

Corresponding author: Fajraoui Ayoub, Ph.D. student, research field: system decision support for the design of multi-spindle drilling casings. specified conditions may be obtained more quickly and the redundancy may be decreased.

This is why growth in terms of compactness, efficiency and reliability, requires designers to use a more concise and efficient design optimization technology. We will show, also, the importance of IT tools that seem to be a better solution to assist designers in their decision making.

\section{Methods Used to Gear Train Design}

A gear train is a set of gear wheels commanding each other. The gears used in this process may be concurrent axes or parallel axes.

From the data we have and the specifications received from the client, the design of the gearbox which has the role of transmitting motion to the various holes, is only possible if we start by sizing upside (sizing gears holes gears up to sizing the main drive gear).

Among the methods used, there is the method of continued fractions.

A number $r$ (reduction ratio) is developed continued fractions when you can write it as (a / b) and:

$$
\mathrm{Z}_{\min }>17 \text { and } \mathrm{Z}_{2}+\mathrm{Z}_{1}<120
$$

This method is not complicated but can give great 
accuracy on the report of gear. It is limited by the assortment of the set of removable wheels accompany the machine and the space offered by the lyre on which are mounted the gears.

The second method is the method of partial results. Quotient is determined by the division method two successive terms of the irreducible fraction. Take the example $i=41 / 100$ and perform the successive divisions of the two terms of the irreducible fraction next available below. We divided the greatest number by the smaller, then the divisor by the remains previously obtained, and so on until one of these divisions provided a rest zero or 1 .

The third method is the method of conjugate fractions.

Some methods of calculating and determining the wheels making up the gear train necessary to perform the work of transmission ratio are insufficient. The new method called "Method conjugate fractions" offers a lot more possibilities to find always a gear train that meets the requirements of the driveline to a simple transmission or group between two parallel shafts [2].

The method is to find a rational approach in which a tolerance reports desired speeds. The numerator and denominator of the fraction in question are factored. The factors thus obtained are automatically using a computer program, combined different wheel sets of variants that can lead to the process of proper gear.

\section{Case Description and Optimization}

In this paper we used three software for the design of the gearbox consider a sump application in (ENAB AlCu4MgTi) Aluminum alloys with $4 \%$ copper and magnesium impurities and for Titanium for a two-stroke engine.

This oil contains Carter 4 holes each $120 \mathrm{~mm}$ in diameter and $7 \mathrm{~mm}$ diameter holes 50 , all these holes will be deemed made on the same plane.

For this product, we use CATIA V5 for design, simulation ADAMS 2013 for the gear train of the engagement, MATLAB algorithm for selection of the optimal solution and Kappa PC who is an expert system for the design support system and the decision for kinematic schema generation. The expert system assists with the design, analysis, calculation and library module of the design of two stages and above gearbox with helical gears.
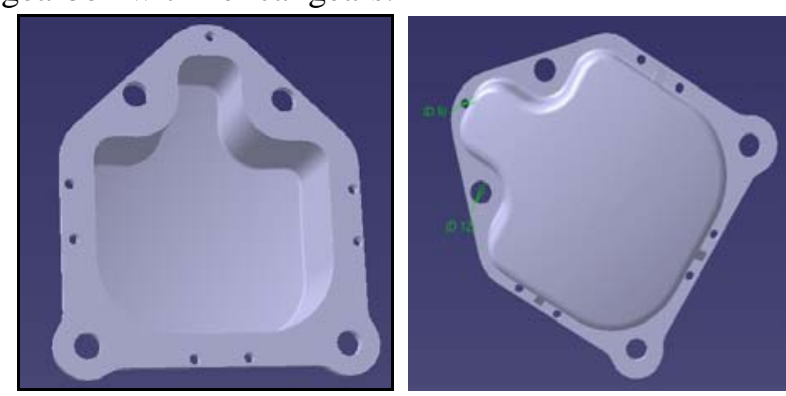

Fig. 1 Carter oil Aluminum ENAB AlCu4MgTi.

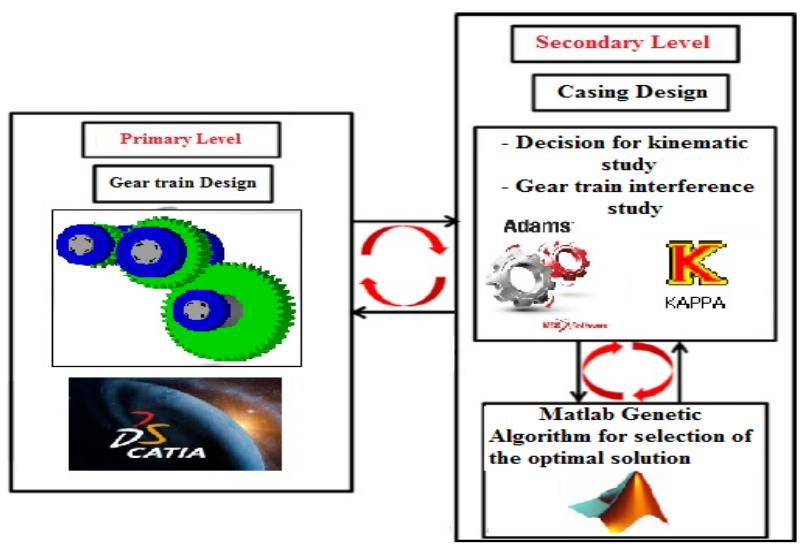

Fig. 2 Flow chart overview of the design phases.

It is noteworthy that each toothed wheel or gear each elementary system is created by the new information. These will be considered by the inference engine in its new warping operation. This chaining phase is summarized in the following chart: data input by the designer.

\subsection{Defining the Structure of Systems (Hierarchy of Classes and Instances)}

In the proposed object-oriented system, classes of objects are arranged as a hierarchy of modular objects with top-down inheritance of slots and methods [3]. Each object is an abstraction of a real-world system component and encapsulates or hides its attributes and behavior from the other objects. Abstraction, encapsulation, modularity, and hierarchy constitute the four fundamental features of a truly object-oriented model. Fig. 3 shows hierarchy of classes and instances. 


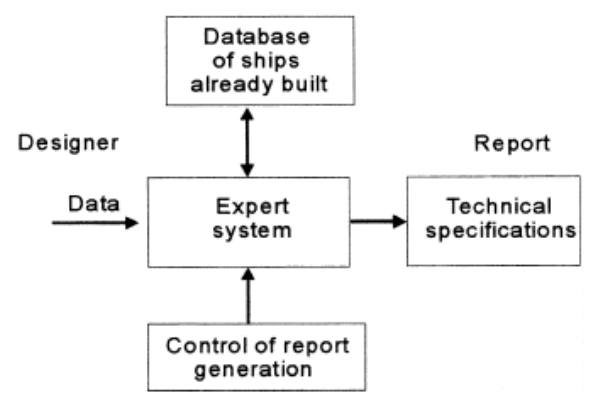

Fig. 3 Objective of using expert system.

The second approach, i.e., object-oriented approach is used for representation of the problem solving knowledge. Of course in this approach, facets and methods are used to solve the problem, as well. The rule set that was developed in the previous section is used by attaching method "Feasibility Rules" to IF-NEEDED facet of "Good candidate" property that loads the rule set and performs environmental feasibility analysis. Fig. 6 shows the details of general class "GEARS". Some proper-ties of objects are listed in this frame as examples [4].

\subsection{Phase 2: Design}

After the knowledge acquisition and representation, the next task is to select the inference technique and control strategy. A prototype system is built to validate the research and to provide guidance for future work. Fig. 4 depicts the general configuration of the proposed system.

The following stages are in the design phase:

(a) Selection of prototype development tool (expert system shell)

There are many expert system shells available. The selection of development tool for gear train must satisfy certain criteria in order to save time and effort in fulfilling its objective. For the present problem, selection must satisfy the following basic conditions:

(1) It must support hybrid knowledge representation techniques;

(2) It must have varied inference facility.

KAPPA-PC expert system generator is mainly composed of a vacuum knowledge base, an inference engine and interface [5]. Using the presented expert system reduces the time during the design and production preparation process. SADBPM is buit

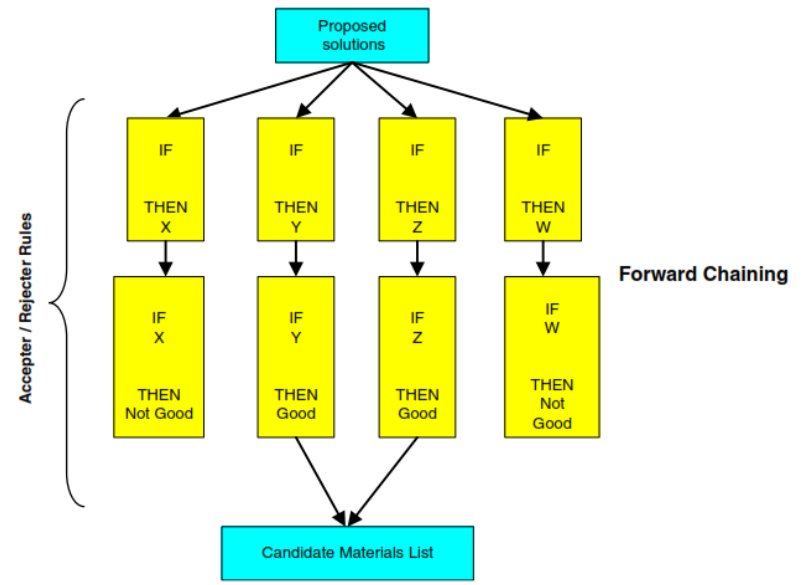

Fig. 4 Principle of forward chaining according to the rules if-then.

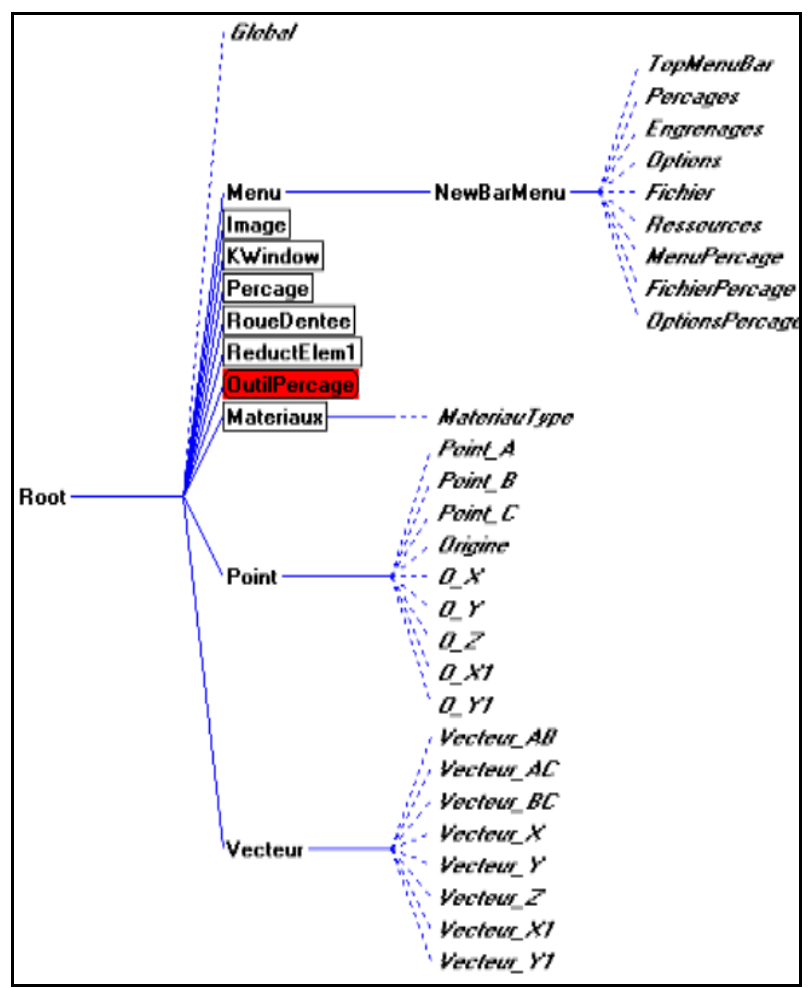

Fig. 5 Oriented object classes of the aided system made by Kappa.

(System Decision Support for the design of a pin hole Case Multiple) with Kappa Kits previously requires to define a schema representation of knowledge in object and object class as well as define the operational knowledge to represent the form of rules and/or methods. Then we will go into Kappa Kits structures and procedures following the proper formalism. Finally, we define the necessary interfaces (menus, windows, etc.). 


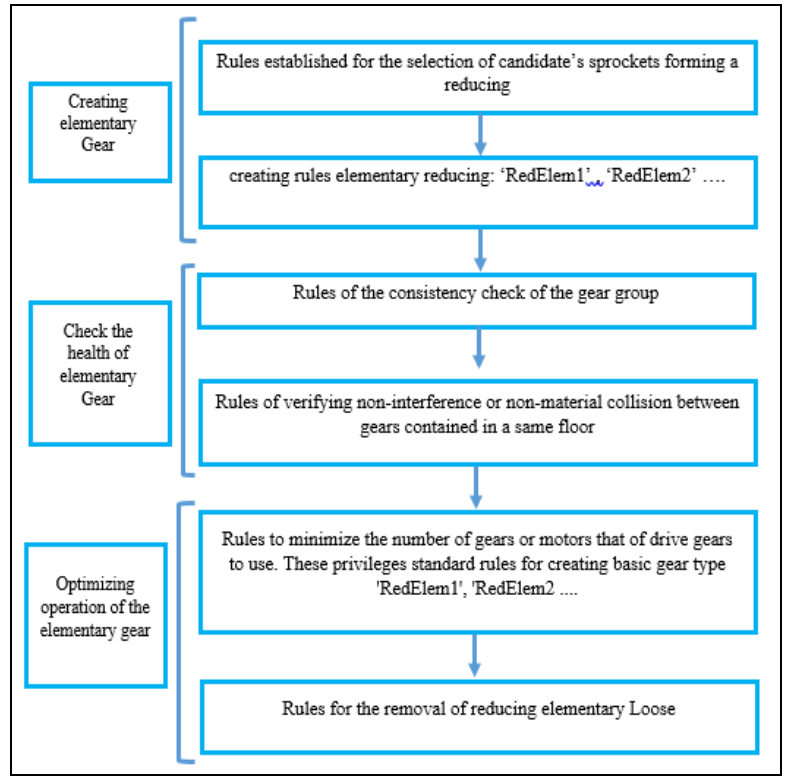

Fig. 6 Ranking rules.

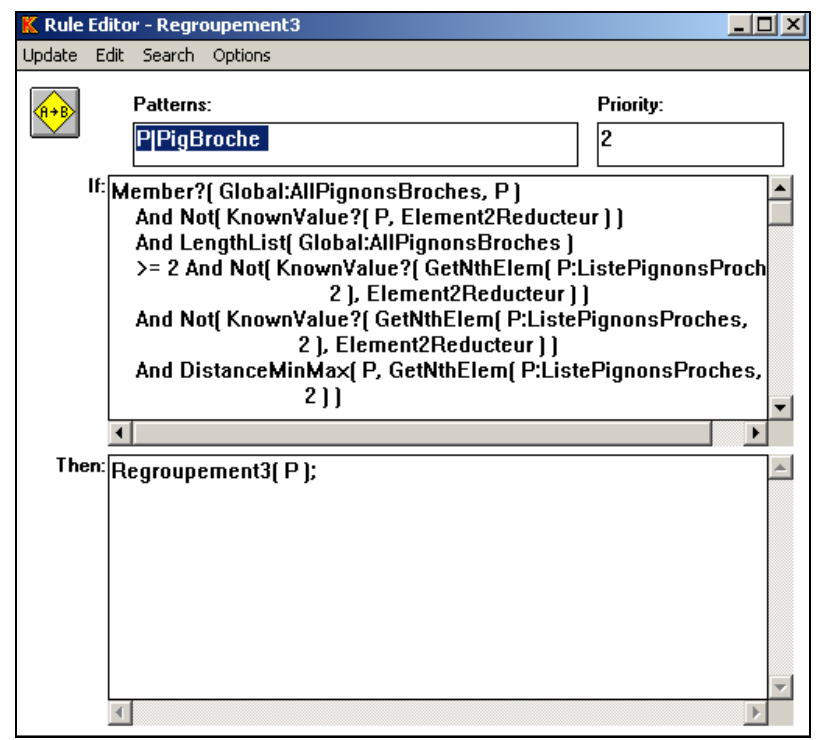

Fig. 7 Example of rule grouping written in Kappa-PC.

Note that in an expert system that previously defined rules can always be changed. We can also enrich the knowledge base by other rules without any modification other parts of the system.

\subsection{Chaining Rules and Creation of Elementary Gear}

Once all drilling object instances are created the program supports chaining rules step through the inference engine [6]. This step, in three phases, the automatic creation of elementary reducing drilling, automatic creation of elementary reducing transmission to finally lead to the creation of the entire drive train housing. The system uses the "forward chaining” in its reasoning mechanism.

\subsection{First Phase Chaining Creation Drilling Reducers}

In this phase, the system starts by associating with each pinion pin creates a list consisting of the other pins gears. This list is arranged in ascending order of their distances from the pin wheel in question. It will be helpful to the rule that selects the pin sprockets together to form an intermediate gear.

Following this operation, the inference engine triggers a candidate rule to create the type of elementary reducing RedElem1, RedElem2 or RedElem3. The creation of a gearbox requires, in parallel, the creation of a toothed wheel type instance "pinions". This wheel has the function of a drive pinion which transmits its motion and power to the receiving wheel. These are the pin gears that have this last function.

Thus, the consistency checks of the combination of gears (engine sprocket wheels and receiver) to form a gear are accomplished through rules. Similarly, a non-coherent will be removed by reducing the outbreak of the rules established for this purpose [7].

Finally, the functional characterization, geometric and dimensional and creates a consistent reduction is achieved by calculation procedures defined in their object classes.

These procedures will be performed after a message transmission by the system. Following this computing operation, a rule verification not for interference between the various gears is automatically started by the inference engine.

\subsection{Second Phase Chaining: Creating Transmission Reducers}

The sizing of this phase is a duplication of the first phase except that the grouping is limited to RedElem2 


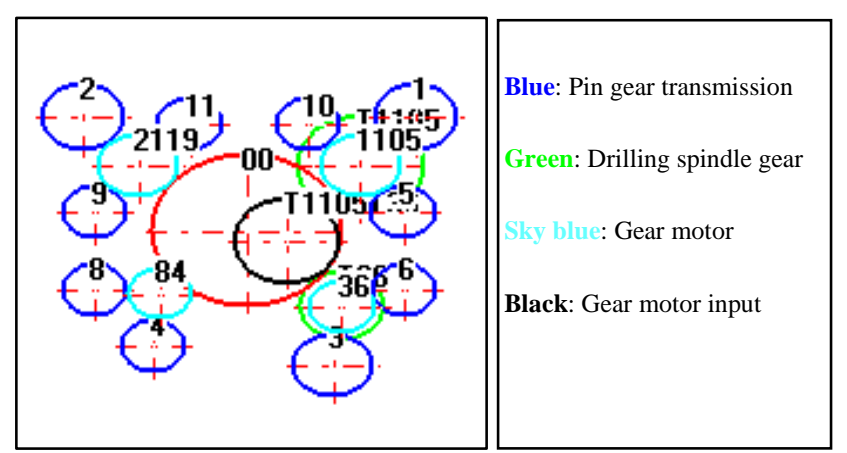

Fig. 8 Second chaining: Chain kinematics drill casing of the oil pan.

type of basic or reducing RedElem1. Fig. 5 shows the results of this second phase chaining for the example given (oil pan).

Because the object-oriented software has no formal module, therefore a method is required to define the calculation of distances so we opted for the creation of an object class "vector" to handle the distances and power create benchmarks [8].

\section{Conclusions}

Once all drilling object instances are created the program supports chaining rules step through the inference engine. This step, in three phases, the automatic creation of elementary reducing drilling, automatic creation of elementary reducing transmission to finally lead to the creation of the entire drive train housing. The system uses the "forward chaining" in its reasoning mechanism.

Since the creation of computer programming phases of the last two types of gear is not yet complete, we will limit ourselves in what follows the presentation of the implementation procedure for the drill gearboxes.

The final results of the kinematic chain of the multi-spindle housing are represented in Fig. 6 which is based on the approach that has been followed for the design of system components and the various methods they used.

The conclusion of this paper has shown that a gearbox is a set of objects organized in one way or another to form the final assembly. We can have several boxes of drawings from the same set of objects. Each object is defined by a set of attributes and a set of methods. These two assemblies are surrounded by a set of functions (procedures) and a set of functional constraints [8].

Thus, in the result of this analysis a conceptual diagram of the gear transmission box was proposed. This scheme formalizes the conceptual analysis that was made. Then the outline of the plan of the integrated system ensuring the conceptual representation of transmission dishes was presented.

\section{References}

[1] Savsani, V., Rao, R. V., and Vakharia, D. P. 2010. "Optimal Weight Design of a Gear Train Using Particle Swarm Optimization and Simulated Annealing Algorithms." Mechanism and Machine Theory 45 (3): 531-41.

[2] Mohamed-Salah, M. E. C. I. B. A. H. 2010. "Modélisation de Calcul des Roues D'échange dans une Transmission Mécanique par Engrenage.” Ph.D thesis, Université Badji Mokhtar de Annaba.

[3] Zeigler, B. P. 2014. Object-oriented simulation with hierarchical, modular models: intelligent agents and endomorphic systems. Academic press.

[4] Zarandi, M. H. F., Mansour, S., Hosseinijou, S. A., and Avazbeigi, M. 2011. “A Material Selection Methodology and Expert System for Sustainable Product Design.” The International Journal of Advanced Manufacturing Technology 57 (9-12) : 885-903.

[5] Rodriguez, B. J. 1997. An Embedded Temporal Expert for Control of a Tandem Accelerator.

[6] Sheremetov, L., Batyrshin, I., Filatov, D., Martinez, J., and Rodriguez, H. 2008. "Fuzzy Expert System for Solving Lost Circulation Problem.” Applied Soft Computing 8 (1): 14-29.

[7] Aziz, E. S. S., and Chassapis, C. 2005. "A Decision-Making Framework Model for Design and Manufacturing of Mechanical Transmission System Development.” Engineering with Computers 21 (2): 164-76.

[8] Savage, M., Coy, J. J., and Townsend, D. P. 1982. "Optimal Tooth Numbers for Compact Standard Spur Gear Sets.” Journal of Mechanical Design 104 (4): 749-57. 